https://doi.org/10.15407/ujpe63.12.1050

A.I. GOMONAI, A.N. GOMONAI

Institute of Electron Physics, Nat. Acad. of Sci. of Ukraine

(21, Universytets'ka Str., Uzhgorod 88017, Ukraine; e-mail: alekgomonai@gmail.com)

\title{
THREE-PHOTON RESONANCE IONIZATION SPECTROSCOPY OF EXCITED EVEN-PARITY STATES OF THE SAMARIUM ATOM
}

\begin{abstract}
Excited even-parity states of the samarium atom in the energy interval 32620-34092 $\mathrm{cm}^{-1}$ have been studied by using single-color three-photon resonance ionization spectroscopy. The energies and total angular momenta of 72 states were determined. Nine new levels were revealed.

Ke ywords: samarium, spectrum, even-parity states, single-color three-photon spectroscopy.
\end{abstract}

\section{Introduction}

Samarium belongs to a group of rare-earth elements with the completely filled external $6 s^{2}$-shell, but with the different occupation degrees of the internal $4 f$ shell. The study of this group of atoms has a fundamental aspect associated with the filling of an internal shell after the external one has been filled, as well as a practical interest owing to numerous applications of those elements in various domains of science and technology.

Despite a rather large number of experimental papers dealing with the samarium atom spectroscopy, the spectrum of atomic energy levels of samarium has not been studied enough. In particular, this is true for bound excited even-parity states. Those states are most interesting from the viewpoint of their practical application in various selective excitation schemes that are used, while solving a wide range of applied problems: the separation of isotopes and the study of their properties, the measurement of ultra low concentrations, high-resolution spectroscopy, and so forth. The overwhelming majority of available data were obtained for even-parity levels of samarium located at energies above $34100 \mathrm{~cm}^{-1}$. This energy interval has been studied rather well making use of

(c) A.I. GOMONAI, A.N. GOMONAI, 2018

1050 two-step (two-color) [1-4, 6, 8-11, 13] and two-photon (single-color) $[5,7,12]$ schemes of level excitation.

Concerning the energies below $34100 \mathrm{~cm}^{-1}$, there are three relevant works $[2,10,13]$. The analysis of two-step schemes of even-parity level excitation that were used in those works testifies that, according to the selection rules [14], the authors of all cited works should observe the excitation of levels with the total angular momenta $J=0 \div 2$. There are 39 levels with $J=0 \div 2$ in the samarium atom within the energy interval $33144-33958 \mathrm{~cm}^{-1}$, common for those works. However, only 29 levels were experimentally detected in work [13], 18 in work [2], and only 2 in work [10]. This fact indicates that the probability of the two-step level excitation strongly depends on the specific excitation scheme.

Taking the aforesaid into account, it is of interest to analyze even-parity states of the samarium atom located at energies below $34100 \mathrm{~cm}^{-1}$, but using an excitation scheme different from those applied in the cited experiments $[2,10,13]$. In this work, which continues our previous researches [7,12], the method of single-color three-photon resonance ionization spectroscopy was used for the determination of the energies and the total angular momenta of excited evenparity states of the samarium atom, which are located in the energy interval $32620-34092 \mathrm{~cm}^{-1}$.

ISSN 2071-0194. Ukr. J. Phys. 2018. Vol. 63, No. 12 


\section{Experimental Part}

The schematic diagram of the experimental installation is shown in Figure. Radiation emitted by a pulsed dye laser FL-2001 (Lambda Physik) pumped by the second harmonic of a Nd:YaG laser was focused into a vacuum chamber making use of a lens (L) with a focal length of $16 \mathrm{~cm}$. In the chamber, radiation intersected at a right angle with a beam of samarium atoms generated by an effusion source. Samarium ions $\mathrm{Sm}^{+}$, which were formed due to the interaction between the atomic and laser beams, were drawn out by appying a constant electric field with a strength of about $100 \mathrm{~V} / \mathrm{cm}$. After having passed through a time-of-flight (TOF) mass spectrometer, the ions were detected by a microchannel particle (MCP) detector of the VEU-7 type. The concentration of samarium atoms in the interaction region was about $10^{10} \mathrm{~cm}^{-3}$. The residual pressure in the vacuum chamber did not exceed $10^{-5} \mathrm{~Pa}$.

The dye phenalemine 160 was used as an active medium. Laser radiation was linearly polarized in the plane parallel to the atomic beam axis. The linear polarization was obtained using a Glan prism (GP). The repetition frequency of laser pulses was $5 \mathrm{~Hz}$. The energy of each pulse was measured with the help of a photo diode (P1) with a calibrated spectral sensitivity. The lasing frequency was determined with an accuracy not lower than $\pm 0.3 \mathrm{~cm}^{-1}$ according to the opto-galvanic spectrum of a $\mathrm{Cu}-\mathrm{Ne}$ hollow cathode lamp (Cu-Ne HCL) and the transmission spectrum of a Fabry-Perot (FP) interferometer, which was registered by a photo diode (P2). All measured signals were sent to the input of a multichannel registration system (RS) and converted into a digital code, which was read out by a computer (PC). The latter controlled the experimental procedure in whole.

Experimentally, we measured the dependence of the yield of singly charged samarium ions on the lasing frequency, $N^{+}(\omega)$, within the interval 15140$17050 \mathrm{~cm}^{-1}$. The radiation line width did not exceed $0.2 \mathrm{~cm}^{-1}$ over the whole examined interval.

Samarium ions $\mathrm{Sm}^{+}$were generated owing to the three-photon ionization. The two-photon excitation of even-parity states manifested itself as resonance peaks of various amplitudes in the dependence $N^{+}(\omega)$. When the lasing frequency $\omega$ was changed, the laser radiation intensity in the interaction region was maintained at a constant level of

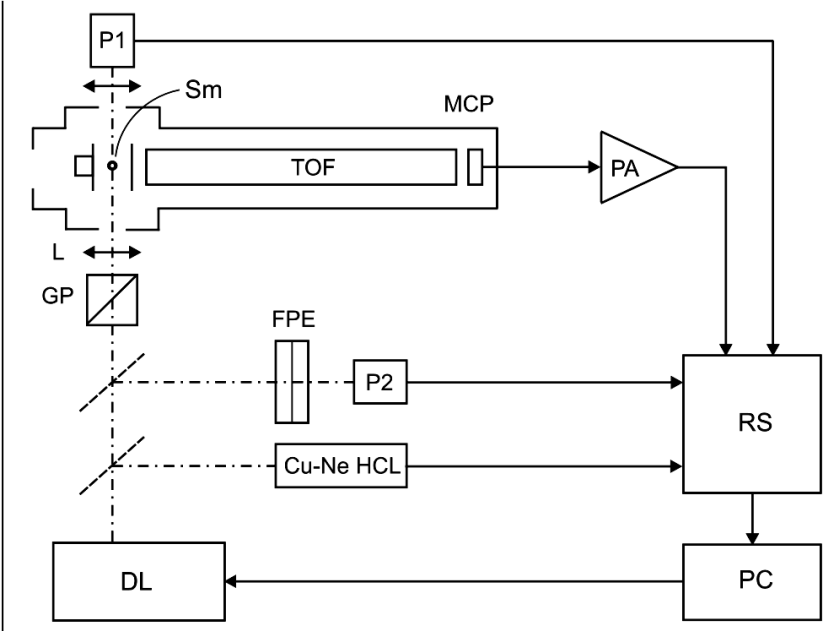

Schematic diagram of the experimental installation: (DL) dye laser, (GP) Glan prism, (L) lens, (Cu-Ne HCL) Cu-Ne hollow cathode lamp, (FPE) Fabri-Perot etalon, (P1,P2) photo diodes, (TOF) time-of-flight mass spectrometer, (MCP) microchannel detector, (PA) preamplifier, (RS) registration system, (PC) computer

$9 \times 10^{7} \mathrm{~W} / \mathrm{cm}^{2}$. This level makes it possible to reveal the majority of peaks associated with two-photon transitions in the dependence $N^{+}(\omega)$ [12].

A method for identifying the resonance structure in three-photon ionization spectra and determining the energies and total angular momenta of even-parity states was described in works $[7,12]$ in detail. Its essence consists in combining the peaks observed in the dependence $N^{+}(\omega)$ into groups associated with the two-photon excitation of the same upper evenparity levels from different lower levels of the main term ${ }^{7} F$.

We recall that the lowest seven even-parity levels of the $4 f^{6} 6 s^{2}$ configuration, which form the ground septet term ${ }^{7} F_{0-6}$, are located in an energy interval from 0 to $4021 \mathrm{~cm}^{-1}$ [16]. Therefore, they all become occupied even at relatively low temperatures. In particular, at an operating temperature of the atomic source of about $900 \mathrm{~K}$, they are occupied in accordance with the Boltzmann distribution as follows: $18 \%\left({ }^{7} F_{0}\right), 35 \%\left({ }^{7} F_{1}\right), 26 \%\left({ }^{7} F_{2}\right), 13 \%\left({ }^{7} F_{3}\right), 5 \%$ $\left({ }^{7} F_{4}\right), 2 \%\left({ }^{7} F_{5}\right)$, and $0.5 \%\left({ }^{7} F_{6}\right)$. Hence, according to the selection rules, a certain level can be occupied owing to transitions from different levels of the ground term ${ }^{7} F$. In particular, in the case of excitation of the samarium atom even-parity states, these are two-photon transitions obeying the selection 
rules: $P_{o}=P$ and $\Delta J=0, \pm 1, \pm 2$, where $P_{o}$ and $P$ are the parities of the ground and excited levels, respectively; and $J$ the total angular momentum. Additionally prohibited are the two-photon transitions $J_{0}=0 \rightarrow J=1$ and $J_{0}=1 \rightarrow J=0$ [14].

The peak frequencies in every group associated with the two-photon excitation of a certain level with the energy $E$ are determined by the relation

$\omega=\frac{E-E_{0}\left(J_{0}\right)}{2}$,

where $E_{0}\left(J_{0}\right)$ is the lowest-level energy of the ground term, from which the transition takes place. These energies are: 0 (0), 292.58 (1), $811.92(2), 1489.55$ (3), 2273.09 (4), 3125.40 (5), and $4020.66 \mathrm{~cm}^{-1}$ (6) [16].

When identifying the levels with $J \geq 1$, only groups containing three or more maxima were analyzed. Note that, in the case of two maxima, the accidental coincidence of the frequencies of observed maxima and the frequencies of possible two-photon transitions is highly probable [7,12]. Groups with two peaks were analyzed only with respect to the excitation of levels with the total angular momentum $J=0$, for which only two-photon transitions from the initial levels ${ }^{7} F_{0}$ and ${ }^{7} F_{2}$ are allowed [14].

Not all possible two-photon transitions allowed by the selection rules manifested themselves as distinct peaks in the dependence $N^{+}(\omega)$ measured at a lasing intensity of $9 \times 10^{7} \mathrm{~W} / \mathrm{cm}^{2}$. In order to reveal such peaks or confirm their absence, the dependence $N^{+}(\omega)$ was measured at various intensities in vicinities of the corresponding frequencies that were determined by relation (1). The lasing intensity was elevated by increasing the pumping intensity and was reduced by changing the position of focusing lens with respect to the exact focus position with the help of a micrometer screw. In the latter case, a decrease in the intensity was accompanied by a growth of the focal volume and, accordingly, an increase in the number of samarium atoms in the interaction region. As a result, the resonance structure revealed itself better at low laser radiation intensities. The lower limit of the laser radiation intensity was governed by the sensitivity threshold of a recording equipment, whereas the upper limit depended on the spectral interval and was determined by the luminescence curve of the dye phenalemine 160 . The maximum intensity of the focused laser radiation in the interaction region did not exceed $5 \times 10^{8} \mathrm{~W} / \mathrm{cm}^{2}$.

\section{Experimental Results and Their Discussion}

The determined energies $E$ and total angular momenta $J$ of excited even-parity levels of the samarium atom, as well as the frequencies of two-photon transitions corresponding to the excitation of those levels from various initial levels of the ground term ${ }^{7} F$, are quoted in Table. The superscript $h$ is used to mark the transitions that were only observed as rather pronounced peaks in the dependence $N^{+}(\omega)$, if the laser radiation intensity exceeded $9 \times 10^{7} \mathrm{~W} / \mathrm{cm}^{2}$.

The total angular momenta $J$ 's of excited levels were determined according to the selection rules [14] and proceeding from the values of the total angular momentum $J_{0}$ of the initial levels, from which twophoton transitions took place. For the excited levels with $J=0 \div 4$, which parameters are quoted in Table, the selection rules are as follows:

$J_{0}=0,2 \rightarrow J=0$

$J_{0}=1 \div 3 \rightarrow J=1$

$J_{0}=0 \div 4 \rightarrow J=2$

$J_{0}=1 \div 5 \rightarrow J=3$

$J_{0}=2 \div 6 \rightarrow J=4$.

Note that Table does not contain columns associated with two-photon transitions from the initial levels ${ }^{7} F_{5}$ and ${ }^{7} F_{6}$, which should be observed if the levels with the total angular momenta $J=3$ and 4 , respectively, were excited, because no such transition were detected. In our opinion, this occurred because of a low probability of those transitions together with a very low population of the ${ }^{7} F_{5}$ and ${ }^{7} F_{6}$ levels.

According to the literature data $[2,10,13]$, there are 64 excited even-parity levels of the samarium atom in our spectral interval. Of those levels, the excitation of 27 levels was observed in work [2], 18 levels in work [10], and 28 levels in work [13]. The energies of corresponding levels determined by the cited authors are also given in Table. A comparison of our energy values with the data of works $[2,10,13]$ testifies to their good agreement. The maximum difference from the data of work [2] does not exceed $0.3 \mathrm{~cm}^{-1}, 0.5 \mathrm{~cm}^{-1}$ from the data of work [10] (No. 71), and $0.6 \mathrm{~cm}^{-1}$ from the data of work [13] (No. 13).

The levels presented in Table can be conditionally divided into two groups. The first group consists of 59 levels for which - except for four ones (these are Nos. 1, 5, 23, and 71) - all possible

ISSN 2071-0194. Ukr. J. Phys. 2018. Vol. 63, No. 12 
Parameters of excited even-parity states of the samarium atom

\begin{tabular}{|c|c|c|c|c|c|c|c|c|c|c|c|c|c|}
\hline \multirow{2}{*}{ No. } & \multicolumn{4}{|c|}{ Energy, $\mathrm{cm}^{-1}$} & \multicolumn{4}{|c|}{$\begin{array}{l}\text { Total angular } \\
\text { momentum }\end{array}$} & \multicolumn{5}{|c|}{$\begin{array}{l}\text { Frequency of two-photon transitions from various } \\
\text { initial levels of the ground term }{ }^{7} \mathrm{~F}, \mathrm{~cm}^{-1}\end{array}$} \\
\hline & $E$ & {$[2]$} & {$[10]$} & {$[13]$} & $J$ & {$[2]$} & {$[10]$} & {$[13]$} & ${ }^{7} F_{0}$ & ${ }^{7} F_{1}$ & ${ }^{7} F_{2}$ & ${ }^{7} F_{3}$ & ${ }^{7} F_{4}$ \\
\hline $1^{\#}$ & 34091.5 & & & & 1,3 & & & & & 16899.6 & 16639.6 & 16301.0 & 15909.3 \\
\hline $2^{\#}$ & 34052.1 & & & & 1 & & & & & 16879.9 & 16620.0 & 16281.2 & \\
\hline 3 & 34041.4 & 34041,3 & & & 3 & 1,3 & & & & 16874.4 & $16614.6^{h}$ & 16276.1 & 15884.2 \\
\hline 4 & 33998.4 & 33998.1 & & & 2 & 2 & & & 16999.2 & 16852.8 & 16593.3 & 16254.5 & 15862.6 \\
\hline 5 & 33975.1 & 33975.1 & & & 3 & 1,3 & & & & 16841.2 & $16581.7^{h}$ & $16242.6^{h}$ & 15851.2 \\
\hline 6 & 33957.7 & 33957.9 & & 33957.5 & 2 & 2 & & $0,1,2$ & 16978.8 & 16832.6 & 16572.9 & 16234.2 & 15842.3 \\
\hline 7 & 33956.2 & 33955.9 & & 33956.0 & 2 & 2 & & $0,1,2$ & 16978.0 & 16831.7 & 16572.2 & 16233.5 & 15841.6 \\
\hline 8 & 33912.6 & & & 33912.6 & 2 & & & $0,1,2$ & 16956.3 & 16810.1 & 16550.4 & $16211.4^{h}$ & 15819.7 \\
\hline 9 & 33894.0 & 33893.7 & & 33893.9 & 2 & 2 & & $0,1,2$ & 16946.9 & 16800.7 & $16541.1^{h}$ & 16202.3 & 15810.5 \\
\hline 10 & 33849.7 & 33849.6 & & 33849.7 & 2 & 2 & & $0,1,2$ & 16924.9 & 16778.6 & 16519.1 & 16179.9 & 15788.3 \\
\hline 11 & 33833.8 & 33833.9 & & 33833.5 & 2 & 2 & & $0,1,2$ & 16916.9 & 16770.7 & 16511.0 & 16172.0 & 15780.3 \\
\hline 12 & 33801.4 & & & 33801.4 & 2 & & & $0,1,2$ & 16900.8 & 16754.4 & 16494.8 & $16155.8^{h}$ & 15764.1 \\
\hline 13 & 33747.3 & 33747.4 & & 33747.9 & 2 & 2 & & $0,1,2$ & 16873.6 & 16727.4 & $16467.6^{h}$ & $16128.9^{h}$ & 15737.2 \\
\hline 14 & 33739.2 & & & 33739.2 & 1 & & & $0,1,2$ & & 16723.3 & 16463.7 & 16124.7 & 15733.1 \\
\hline $15^{*}$ & 33736.1 & 33736.4 & & & 1 & 1 & & & $\underline{16868.2}$ & 16721.8 & 16462.0 & 16123.2 & $\underline{15731.4}$ \\
\hline 16 & 33734.2 & 33734.3 & & & 2 & 2 & & & 16867.0 & 16720.9 & 16461.1 & 16122.3 & 15730.6 \\
\hline $17^{*}$ & 33702.6 & 33702.7 & & & 1 & 1 & & & $\underline{16851.4}$ & 16705.1 & $16445.3^{h}$ & $16106.5^{h}$ & \\
\hline 18 & 33645.3 & 33645.4 & 33645.6 & 33645.6 & 1 & 1 & 1 & $0,1,2$ & & 16676.3 & $16416.8^{h}$ & $16077.8^{h}$ & \\
\hline 19 & 33630.7 & 33630.5 & & & 2 & 2 & & & 16815.3 & 16669.2 & 16409.3 & $16070.4^{h}$ & 15678.9 \\
\hline 20 & 33627.1 & 33627.1 & & & 2 & 2 & & & 16813.6 & 16667.2 & 16407.6 & 16068.7 & 15677.0 \\
\hline $21^{\#}$ & 33609.9 & & & & 1 & & & & & 16658.7 & 16399.1 & 16060.0 & \\
\hline $22^{\#}$ & 33608.1 & & & & 1 & & & & & 16657.7 & 16398.0 & 16059.4 & \\
\hline 23 & 33607.4 & 33607.1 & & & 3 & 1,3 & & & & 16657.3 & 16397.8 & 16059.0 & 15667.2 \\
\hline 24 & 33588.2 & & & 33588.1 & 1,2 & & & $0,1,2$ & & $16647.7^{h}$ & $16388.1^{h}$ & $16049.5^{h}$ & \\
\hline 25 & 33585.5 & 33585.3 & & 33585.2 & 2 & 2 & & $0,1,2$ & 16792.7 & 16646.5 & 16386.8 & $16047.9^{h}$ & 15656.2 \\
\hline 26 & 33541.5 & & & 33541.2 & 0 & & & $0,1,2$ & 16770.7 & & 16364.8 & & \\
\hline 27 & 33526.2 & & & 33526.2 & 1 & & & 1 & & 16616.9 & $16357.1^{h}$ & 16018.2 & \\
\hline 28 & 33522.0 & & & 33522.1 & 1 & & & 1 & & $16614.6^{h}$ & $16355.1^{h}$ & $16016.3^{h}$ & \\
\hline 29 & 33507.7 & 33507.5 & & & 1 & 1,3 & & & & $16607.5^{h}$ & 16348.1 & $16008.9^{h}$ & \\
\hline 30 & 33502.0 & & & 33502.0 & 1 & & & 1 & & 16604.8 & $16345.0^{h}$ & $16006.1^{h}$ & \\
\hline 31 & 33463.1 & & & 33462.9 & 1 & & & 1 & & 16585.3 & $16325.5^{h}$ & $15986.8^{h}$ & \\
\hline 32 & 33433.8 & & & 33433.5 & 1 & & & 1 & & 16570.6 & $16310.9^{h}$ & $15972.2^{h}$ & \\
\hline $33^{a}$ & 33397.0 & & 33396.9 & & 3 & & 3 & & & & & 15953.7 & 15562.0 \\
\hline 34 & 33393.5 & & & 33393.3 & 1 & & & 1 & & 16550.4 & $16290.8^{h}$ & $15952.0^{h}$ & \\
\hline 35 & 33377.9 & 33377.6 & & & 2 & 2 & & & 16689.0 & 16542.7 & 16283.2 & 15944.1 & 15552.3 \\
\hline 36 & 33374.6 & & & 33374.5 & 1 & & & 1 & & $16541.0^{h}$ & $16281.2^{h}$ & $15942.6^{h}$ & \\
\hline 37 & 33364.8 & & & 33364.8 & 1 & & & 1 & & 16536.2 & 16276.3 & $15937.6^{h}$ & \\
\hline 38 & 33334.2 & & & 33334.2 & 1 & & & 1 & & 16520.9 & $16261.2^{h}$ & $15922.2^{h}$ & \\
\hline $39^{*}$ & 33328.7 & & 33328.9 & 33329.1 & 1 & & 1 & 1 & $\underline{16664.4}$ & 16518.1 & 16258.3 & 15919.5 & $\underline{15527.8}$ \\
\hline $40^{*}$ & 33326.3 & 33326.2 & & & 0 & 0 & & & $\overline{16663.1}$ & $\underline{16516.6}$ & $16257.2^{h}$ & $\underline{15918.5^{h}}$ & $\underline{15526.7}$ \\
\hline 41 & 33325.7 & & & 33325.6 & 1 & & & 1 & & 16516.4 & $16256.9^{h}$ & $15918.2^{h}$ & \\
\hline 42 & 33306.5 & 33306.4 & & & 2 & 2 & & & 16653.3 & 16506.9 & 16247.3 & $15908.4^{h}$ & 15516.7 \\
\hline $43^{a}$ & 33288.6 & & 33288.5 & & 3 & & 3 & & & 16498.1 & 16238.2 & & 15507.8 \\
\hline
\end{tabular}


Continuation of Table

\begin{tabular}{|c|c|c|c|c|c|c|c|c|c|c|c|c|c|}
\hline \multirow{2}{*}{ No. } & \multicolumn{4}{|c|}{ Energy, $\mathrm{cm}^{-1}$} & \multicolumn{4}{|c|}{$\begin{array}{l}\text { Total angular } \\
\text { momentum }\end{array}$} & \multicolumn{5}{|c|}{$\begin{array}{l}\text { Frequency of two-photon transitions from various } \\
\text { initial levels of the ground term }{ }^{7} F, \mathrm{~cm}^{-1}\end{array}$} \\
\hline & $E$ & {$[2]$} & [10] & [13] & $J$ & {$[2]$} & [10] & [13] & ${ }^{7} F_{0}$ & ${ }^{7} F_{1}$ & ${ }^{7} F_{2}$ & ${ }^{7} F_{3}$ & ${ }^{7} F_{4}$ \\
\hline 44 & 33278.8 & 33278.6 & & & 2 & 2 & & & 16639.3 & 16493.2 & 16233.5 & 15894.6 & 15502.9 \\
\hline $45^{a}$ & 33251.7 & & 33251.5 & & 3 & & 3 & & & 16479.6 & & & 15489.3 \\
\hline 46 & 33241.0 & & & 33240.9 & 1 & & & 1 & & 16474.2 & $16214.5^{h}$ & 15875.8 & \\
\hline 47 & 33223.6 & & & 33223.7 & 1 & & & 1 & & 16465.6 & 16205.7 & $15867.0^{h}$ & \\
\hline 48 & 33198.4 & & & 33198.3 & 1 & & & 1 & & $16452.8^{h}$ & 16193.4 & $15854.3^{h}$ & \\
\hline $49^{a}$ & 33186.8 & & 33186.7 & & 4 & & 4 & & & & 16187.5 & 15848.6 & \\
\hline $50^{a}$ & 33162.1 & & 33161.9 & & 4 & & 4 & & & & 16175.1 & & 15444.5 \\
\hline 51 & 33158.0 & & & 33157.8 & 1 & & & 1 & & 16432.7 & $16172.9^{h}$ & $15834.3^{h}$ & \\
\hline 52 & 33144.3 & & & 33144.1 & 1 & & & 1 & & 16426.0 & 16166.1 & $15827.3^{h}$ & \\
\hline $53^{*}$ & 33116.8 & 33116.7 & 33117.0 & & 1 & 1 & 1 & & $\underline{16558.4^{h}}$ & 16412.2 & 16152.5 & $15813.6^{h}$ & $\underline{15421.8}$ \\
\hline $54^{a}$ & 33111.0 & & 33110.7 & & 3 & & 3 & & & 16409.1 & & 15810.7 & $\overline{15419.0}$ \\
\hline $55^{a}$ & 33068.0 & & 33067.8 & & 4 & & 4 & & & & 16128.1 & 15789.2 & \\
\hline 56 & 33053.2 & 33053.1 & & & 2 & 2 & & & $16526.5^{h}$ & 16380.4 & $16120.6^{h}$ & $15781.8^{h}$ & 15390.1 \\
\hline 57 & 33035.6 & 33035.7 & & & 2 & 2 & & & 16517.7 & 16371.7 & 16112.0 & $15773.1^{h}$ & 15381.0 \\
\hline 58 & 32995.9 & 32995.9 & & & 0 & 0 & & & 16498.1 & & $16091.8^{h}$ & & \\
\hline $59^{a}$ & 32988.4 & & 32988.4 & & 4 & & 4 & & & & & 15749.4 & 15357.7 \\
\hline 60 & 32985.4 & & 32985.2 & & 1 & & 1 & & & 16346.4 & 16086.8 & 15747.9 & \\
\hline $61^{a}$ & 32973.2 & & 32972.9 & & 1 & & 1 & & & 16340.3 & & 15741.8 & \\
\hline 62 & 32959.2 & 32958.9 & & & 0 & 0 & & & 16479.6 & & 16073.6 & & \\
\hline $63^{a}$ & 32882.3 & & 32882.0 & & 3 & & 3 & & & & 16035.2 & 15696.4 & \\
\hline $64^{\#}$ & 32777.1 & & & & 2 & & & & 16388.6 & 16242.3 & 15982.5 & 15643.6 & 15252.2 \\
\hline $65^{a}$ & 32768.0 & & 32767.9 & & 4 & & 4 & & & & 15978.1 & 15639.2 & \\
\hline $66^{\#}$ & 32736.9 & & & & 2 & & & & 16368.6 & 16222.2 & $15962.4^{h}$ & 15623.6 & 15231.9 \\
\hline $67 \#$ & 32728.5 & & & & 2 & & & & $16364.3^{h}$ & 16217.8 & 15958.2 & 15619.6 & 15227.7 \\
\hline $68^{a}$ & 32727.8 & & 32727.7 & & 3 & & 3 & & & 16217.5 & & 15619.2 & 15227.4 \\
\hline $69^{a}$ & 32709.5 & & 32709.3 & & 4 & & 4 & & & & 15948.7 & 15610.1 & \\
\hline $70^{\#}$ & 32652.4 & & & & 2 & & & & 16326.0 & 16179.9 & 15920.2 & 15581.4 & 15189.9 \\
\hline 71 & 32630.7 & & 32630.2 & & 3 & & 3 & & & $16169.2^{h}$ & 15909.3 & 15570.5 & 15178.8 \\
\hline $72^{\#}$ & 32620.3 & & & & 1 & & & & & $16163.8^{h}$ & 15904.2 & 15565.4 & \\
\hline
\end{tabular}

As for ${ }^{*},{ }^{*},{ }^{a}$, and ${ }^{h}$, see the explanation in the text.

two-photon transitions associated with their excitation were detected. This fact allowed us to unambiguously determine the total angular momenta of those levels. For levels 1, 5, and 23, as was marked above, we did not manage to detect peaks associated with their two-photon excitation from the initial ${ }^{7} F_{5}$ level. In the case of level 71 , the frequency of the allowed two-photon transition from the initial ${ }^{7} F_{5}$ level $\left(14752.7 \mathrm{~cm}^{-1}\right)$ goes beyond the examined spectral interval $15140-17050 \mathrm{~cm}^{-1}$. Nevertheless, the presence of two-photon transitions from the initial lev- els ${ }^{7} F_{1}-{ }^{7} F_{4}$ and the absence of a transition from the initial level ${ }^{7} F_{0}$ onto the indicated four levels enable us to assert that, according to the selection rules $\left(J_{0}=1 \div 5 \rightarrow J=3\right)$, the total angular momentum of those levels equals $J=3$.

Note that Table exhibits two possible values of the total angular momentum, $J=1$ and 3 , for level 1. This occurs because the two-photon transition ${ }^{7} F_{4} \rightarrow E=34091.5$ (level 1) coincides with the twophoton transition ${ }^{7} F_{2} \rightarrow E=32630.7$ (level 59) by the frequency $\left(15909.3 \mathrm{~cm}^{-1}\right)$. The shape of the cor- 
responding peak observed in the dependence $N^{+}(\omega)$ does not allow to make an unambiguous conclusion about whether this maximum is a manifestation of both indicated transitions or only one transition, namely, ${ }^{7} F_{2} \rightarrow E=32630.7$. Since the occupation number of the ${ }^{7} F_{2}$ level is 5.2 times higher than that of the ${ }^{7} F_{4}$ level, the main contribution to the peak amplitude is given, in our opinion, by the two-photon transition from the ${ }^{7} F_{2}$ level. It is evident that the total angular momentum of the considered level will be equal to $J=3\left(J_{0}=1 \div 5 \rightarrow J=3\right)$, if the transition ${ }^{7} F_{4} \rightarrow E=34091.5\left(15909.3 \mathrm{~cm}^{-1}\right)$ does take place, and to $J=1\left(J_{0}=1 \div 3 \rightarrow J=1\right)$ otherwise.

Table also exhibits two possible values of the total angular momentum for level $24, J=1$ and 2 . The presence of transitions from the initial levels ${ }^{7} F_{1}-{ }^{7} F_{3}$ and the absence of transitions from the initial levels ${ }^{7} F_{0}$ and ${ }^{7} F_{4}$ testify that the total angular momentum of this excited level equals $J=1$. However, the transitions from the initial levels ${ }^{7} F_{1}-{ }^{7} F_{3}$ reveal themselves in the dependence $N^{+}(\omega)$ only at high laser radiation intensities exceeding $9 \times 10^{7} \mathrm{~W} / \mathrm{cm}^{2}$. But the amplitudes of corresponding peaks are small at that (a factor of 0.1-0.2 with respect to the maximums of peak amplitudes at a given lasing intensity). For this reason, it is difficult to assert unequivocally that the absence of transitions from the levels ${ }^{7} F_{0}$ and ${ }^{7} F_{4}$ is associated with the fact that they are forbidden by the selection rules $\left(J_{0}=1 \div 3 \rightarrow J=1\right)$ rather than because of their low probability. It is evident that, in the latter case, the total angular momentum of the considered level would be equal to $J=2$.

The second group of levels includes 13 levels, for which we did not manage to identify all possible twophoton transitions associated with their excitation (in Table, they are marked by the superscript $a$ ). In particular, only two transitions were observed for 10 levels (Nos. 33, 45, 49, 50, 55, 59, 61, 63, 65, and 69). For other three levels (Nos. 43, 54, and 68), three transitions were observed, but the corresponding groups were "split". For instance, in the case of level 43, transitions from the initial levels ${ }^{7} F_{1},{ }^{7} F_{2}$, and ${ }^{7} F$ were detected, but no transition from the level ${ }^{7} F_{3}$. In the case of levels 54 and 68, transitions from the levels ${ }^{7} F_{1},{ }^{7} F_{3}$ and ${ }^{7} F_{4}$ were observed, but the transition from the level ${ }^{7} F_{2}$ was absent. As a result, those levels were not identified following the method described above, namely, the combination of observed transitions into groups corresponding to the excitation of the same upper levels from different initial levels of the ground term ${ }^{7} F$. The corresponding transitions were identified on the basis of data of work [10]. The values of the total angular momentum for those levels, which are quoted in Table, were also taken from work [10].

A comparison of our values obtained for the total angular momentum with the data of works $[2,10,13]$ shows that they are in good agreement, but with a few exceptions. In particular, the $J$-values are identical for 36 levels. For nine levels, our $J$-values agree with the set of possible values proposedfor the total angular momentum in works [2, 13]. Five levels-Nos. 15, 17, 39, 40, and 53-are exceptions; they are marked by the superscript ${ }^{*}$ in Table. They demonstrate the transitions from the initial levels ${ }^{7} F_{0}-{ }^{7} F_{4}$ (Nos. 15 , 39,40 , and 53) and ${ }^{7} F_{0}-{ }^{7} F_{3}$ (No. 17), which, according to the selection rules $\left(J_{0}=0 \div 4 \rightarrow J=2\right)$, means that the total angular momentum of those levels equals $J=2$. However, this value does not coincide with the data of works $[2,10]$ (see Table). The fact that the experiments in works [2] and [10], where different two-step schemes to excite level 53 were used, gave the same value of its total angular momentum, $J=1$, testifies to an accidental coincidence of the frequencies of peaks detected in the dependence $N^{+}(\omega)$ with the frequencies of the two-photon transitions ${ }^{7} F_{0} \rightarrow 33116.8\left(\omega=16558.4 \mathrm{~cm}^{-1}\right)$ and ${ }^{7} F_{4} \rightarrow$ $33116.8\left(\omega=15421.8 \mathrm{~cm}^{-1}\right)$. Those transitions are underscored in Table. An insignificant difference between our energy value of level $53\left(33116.8 \mathrm{~cm}^{-1}\right)$ and the data of works [2] $\left(33116.7 \mathrm{~cm}^{-1}\right)$ and [10] $\left(33117.0 \mathrm{~cm}^{-1}\right)$ evidences that, most likely, we deal with the excitation of the same level. For this reason, Table gives the value of the total angular momentum for this level, $J=1[2,10]$. In our opinion, a similar situation takes place in the case of levels 15, 17, 39, and 40. Therefore, the values of $J$ taken from work [2] are also indicated for them in Table, and the "accidental" transitions are underscored.

We revealed nine new even-parity states of the samarium atom. There are no data about them in the literature. In Table, they are marked by the superscript \#. According to the selection rules [14], the excitation of all those levels could also have been observed in work [10], four levels (Nos. 1, 2, 21, and 22 ) in work Jay [2], and two levels (Nos. 21 and 22) in work [13]. However, the authors of the indicated works did not detect those levels. In our opinion, this 
fact is associated with a low probability of their twostep excitation in the framework of the schemes used in works [2, 10, 13].

At the same time, we did not succeed in detecting the two-photon transitions associated with the excitation of the level $E=33530.8 \mathrm{~cm}^{-1}, J=0$ [10] from the initial levels ${ }^{7} F_{0}\left(\omega=16765.4 \mathrm{~cm}^{-1}\right)$ and ${ }^{7} F_{2}\left(\omega=16359.4 \mathrm{~cm}^{-1}\right)$. Most likely, this is a result of a low probability of the indicated transitions.

\section{Conclusions}

Excited even-parity states of the samarium atom have been studied, by using the single-color three-photon resonance ionization spectroscopy method. The energies and total angular momenta of 72 states within the energy interval $32620-34092 \mathrm{~cm}^{-1}$ were determined. Nine new levels were found, the data on which are absent from the literature.

A comparison of the results of our work with the data of works $[2,10,13]$, which were obtained with the use of other excitation schemes of those levels, demonstrates their good agreement. The maximum difference between the energy values obtained in our experiment and in works $[2,10,13]$ does not exceed $0.3,0.5$, and $0.6 \mathrm{~cm}^{-1}$, respectively.

The exact total angular momenta were determined for 53 levels. Our $J$-values obtained for 36 levels coincide with the data of works $[2,10,13]$, and the results for 9 levels are completely consistent with the set of possible values proposed in works $[2,13]$.

Within the energy interval $33144.3-33957.7 \mathrm{~cm}^{-1}$, which is common for our work and works [2, 10, 13], and where the levels with $J==0 \div 2$ are located, we observed the excitation of 41 levels; the excitation of 18 levels was observed in work [2], 2 levels in work [10], and 29 level in work [13]. Thus, the advantages of the method of single-color three-photon resonance ionization spectroscopy also include a possibility of detecting, in the same experiment, a maximum possible number of excited even-parity levels, two-photon transitions into which are allowed by the selection rules.

1. L. Jia, C. Jing, Z. Zhou, F. Lin. Studies of high-lying levels of Sm I: energies and isotope shifts. J. Opt. Soc. Am. B 10, 1317 (1993).

2. T. Jayasekharan, M.A.N. Razvi, G.I. Bhale. Observation of new even-parity states of Sm I by resonance ionization mass spectrometry. J. Opt. Soc. Am. B 13, 641 (1996).
3. H. Park, H. Kim, J. Yi, J. Han, J. Lee. Resonant threephoton photoionization of Sm atoms. J. Korean Phys. Soc. 30, 453 (1997).

4. T. Jayasekharan, M.A.N. Razvi, G.L. Bhale. Investigation of new high-lying even-parity energy levels of the samarium atom below its first ionization limit. J. Opt. Soc. Am. B 17, 1607 (2000).

5. A.I. Gomonai, O.I. Plekan. Single-colour resonance threephoton ionization of samarium atoms. J. Phys. B 36, 4155 (2003).

6. A.K. Pulhani, M.I. Shah, V. Dev, B.M. Suri. High-lying even-parity excited levels of atomic samarium. J. Opt. Soc. Am. B 22, 1117 (2005).

7. O.I. Gomonai. Single-color three-photon spectroscopy of excited samarium atoms. Ukr. Fiz. Zh. 53, 12 (2008) (in Ukrainian).

8. Y. Zhao, C. Dai, F. Guan. Photoionization spectra of an Sm atom with stepwise excitation J. Phys. B 42, 1 (2009).

9. W. Qin, C. Dai, Y. Xiao, H. Zhao. Experimental study of highly excited even-parity bound states of the Sm atom. Chin. Phys. B 18, 3384 (2009).

10. Y. Zhao, C. Dai, S. Ye. Study on even-parity highly excited states of the Sm atom. J. Phys. B 44, 195001 (2011).

11. M. Li, C. Dai, J. Xie. Even-parity states of the Sm atom with stepwise excitation. Chin. Phys. B 20, 063204 (2011).

12. A.I. Gomonai, E.Yu. Remeta. Research of highly excited states of samarium atom. Opt. Spectrosk. 112, 17 (2012) (in Russian).

13. A.U. Seema, P.K. Mandal, A.D. Rath, V. Dev. New evenparity high-lying levels of Sm I and measurement of isotope shifts by two-color resonance ionization mass spectrometry. J. Quant. Spectrosc. Radiat. Transfer. 145, 197 (2014).

14. K.D. Bonin, T.J. Mcllrath. Two-photon electric-dipole selection rules. J. Opt. Soc. Am. B 1, 52 (1984).

15. A.I. Gomonay, E.Yu. Remeta. Field strength effect on the resonance structure of three-photon ionization spectra of samarium atom. Opt. Spectrosk. 114, 329 (2013) (in Russian).

16. N.C. Martin, R. Zalubas, L. Hagan. Atomic Energy Levels: The Rare-Earth Elements, NSRDS-NBS-60 (US Gov. Print. Office, Washington, DC, 1978).

Translated from Ukrainian by O.I. Voitenko

\section{О.І. Гомонай, Г.М. Гомонай}

ТРИФОТОННА РЕЗОНАНСНО-ІОНІЗАЦІЙНА СПЕКТРОСКОПІЯ ЗБУДЖЕНИХ ПАРНИХ СТАНІВ ATOMA CAMAPIЮ

Р е $з$ ю м е

Методом одноколірної трифотонної резонансно-іонізаційної спектроскопіі досліджено збуджені парні стани атома самарію, розташовані в області енергій 32620-34092 см ${ }^{-1}$. Визначено енергії та повні кутові моменти 72 станів. Виявлено 9 нових рівнів, дані про які відсутні в літературі. 